\title{
Ubiquitous overexpression of a transgene encoding the extracellular portion of the Drosophila Roughest-Irregular Chiasm C protein induces early embryonic lethality
}

\author{
LIVIA MODA ${ }^{1}$, RICARDO C. MACHADO ${ }^{2}$ and RICARDO GUELERMAN P. RAMOS $^{1 *}$ \\ ${ }^{1}$ Departamento de Morfologia, Faculdade de Medicina de Ribeirão Preto, \\ Universidade de São Paulo - 14049-900 Ribeirão Preto, São Paulo, Brazil \\ ${ }^{2}$ Programa de Biologia Molecular, Instituto de Biofísica Carlos Chagas Filho \\ Centro de Ciências da Saúde, Universidade Federal do Rio de Janeiro \\ 21949-000 Rio de Janeiro, RJ, Brazil
}

Manuscript received on May 5, 2000; accepted for publication on May 22, 2000;

presented by LENY A. CAVALCANTE

\begin{abstract}
The cell adhesion molecule Rst-irreC is a transmembrane glycoprotein of the immunoglobulin superfamily involved in several important developmental processes in Drosophila, including axonal pathfinding in the optic lobe and programmed cell death and pigment cell differentiation in the pupal retina. As an initial step towards the "in vivo" functional analysis of this protein we have generated transgenic fly stocks carrying a truncated cDNA construct encoding only the extracellular domain of Rst-IrreC under the transcriptional control of the heat shock inducible promoter hsp70. We show that heat-shocking embryos bearing the transgene during the first $8 \mathrm{hs}$ of development lead to a 3-4 fold reduction in their viability compared to wild type controls. The embryonic lethality can already be produced by applying the heat pulse in the first $3 \mathrm{hs}$ of embryonic development, does not seem to be suppressed in the absence of wildtype product and is progressively reduced as the heat treatment is applied later in embryogenesis. These results are compatible with the hypothesis of the lethal phenotype being primarily due to heterophilic interactions between Rst-IrreC extracellular domain and an yet unknown ligand.
\end{abstract}

key words: Drosophila, transgenic organisms, cell adhesion, immunoglobulin superfamily.

\section{INTRODUCTION}

The development of an organism involves a relatively small number of evolutionarily conserved cross interacting gene pathways organized in a loose hierarchy, whose differential activation, in different developmental contexts, are critical for the final outcome of the process. The ability of a protein to correctly perform its function during development is therefore highly dependent not only on the integrity

Correspondence to: Ricardo Guelerman Pinheiro Ramos E-mail: rgpramos@fmrp.usp.br of its functional domains of but also on the correct spatial and temporal distribution of its interacting partners. By disturbing this delicate balance through "in vivo" manipulation of gene expression patterns, together with the detailed phenotypic characterization of transgenic organisms carrying specially engineered versions of genes of interest, important information on the molecular circuitry they participate can be generated, while at the same time identifying which structural domains of their protein products are essential for proper functioning. 
The roughest irregular chiasmC (rst-irreC) locus is a pleiotropic genetic function mapping in the $\mathrm{X}$ chromosome, essential for a number of developmental processes in Drosophila melanogaster, including axonal pathfinding in the optic lobe and bristle differentiation as well as programmed cell death during the final steps of compound eye morphogenesis (Boschert et al. 1990, Wolf \& Ready 1991, Ramos et al. 1993, Reiter et al. 1996). It encodes a transmembrane glycoprotein with cell-adhesion and cell recognition characteristics, including five immunoglobulin-like (Ig-like) domains in its extracellular portion and a long cytoplasmic tail containing several sequences of possible functional significance (Ramos et al. 1993, Schneider et al. 1995). The gene is developmentally regulated: Gene transcription starts around embryonic late stage 8 (approximately $3: 30-4 \mathrm{hs}$ of development at $25^{\circ} \mathrm{C}$ ) and continues in a highly dynamic pattern throughout most of embryogenesis. Intriguinly, despite this highly regulated and specific expression pattern at the mRNA level, no obvious embryological defects can be observed in mutants lacking the rst-irreC gene. Almost no expression can be detected during the first two larval stages but high mRNA levels are attained again in late larval-early pupal stages specially in the imaginal discs and in the visual system.

The diversity of developmental processes in which rst-irreC seems to play a significant role makes it a useful tool for genetically and molecularly dissecting them. Additionally, since the main structural features of its protein product are shared by several vertebrate molecules of developmental importance, it is likely that at least some of the genes it interacts with may belong to evolutionary conserved pathways.

As a first step towards an "in vivo" dissection of rst-irreC function we report in this study the construction of cDNA version of its transcript encoding the complete extracellular domain, but lacking the transmembrane and intracellular portions of the wild type protein. This construct was put under control of a heat shock inducible promoter and introduced into Drosophila germline, allowing us to generate multiple stable transgenic lines. We show that generalized overexpression of this extracellular domainonly construct during embryogenesis is sufficient to drastically reduce viability. This effect is particularly intense when embryos are heat shocked in the first half of embryogenesis and can already be observed when transgene expression is induced within the first 3 hours of development, a stage where endogenous Rst-IrreC is not yet present. This latter observation is suggestive that heterophilic interactions, rather than direct interference with the wild type product, may be the cause of embryonic lethality.

\section{MATERIAL AND METHODS}

\section{Maintenance and Manipulation of}

\section{DrosophILA STOCKS}

Flies were normally kept at $25^{\circ} \mathrm{C}$ in $80 \mathrm{~mm} \times 20 \mathrm{~mm}$ vials containing standard fly food medium (Lewis 1960). These were also the conditions for the genetic crosses. Unless otherwise noted, a complete account of the mutant phenotypes mentioned in this study can be found in Lindsley and Zimm (1992). Embryo collection was performed by allowing adult individuals with the appropriate genotype to lay eggs onto $60 \mathrm{~mm}$ petri dishes containing grapejuice collecting medium (Elgin \& Miller 1980).

\section{Heat Shock Treatment}

Embryos (150-250) were collected in grapejuice plates for 1-2 hs, let develop at $25^{\circ} \mathrm{C}$ until the desired stage was reached and then incubated in a waterbath at $30^{\circ} \mathrm{C}$ inside a prewarmed $500 \mathrm{ml}$ beaker for the required time.

\section{CONSTRuCtion of $p C a 18 Z \Delta 3.1$}

The generation of a cDNA encoding only the extracellular portion of the rst-irreC gene product was described in detail in Machado (1997). Briefly, a 714 bp PCR amplified DNA fragment coding for residues 291-529 of Rst-IrreC was cloned into pCa18, a derivative of P-element transformation 
vector pCaSpeR-hs already containing the coding region for residues $1-290$. The resulting $1.19 \mathrm{~kb}$ insert generates a 529 amino acid polypeptide containing the complete extracellular portion of Rst-IrreC plus 4 residues (VNSS) at its carboxyl terminus. Except for these last additions and an amino acid change $(\mathrm{Y} \rightarrow \mathrm{C})$ at portion 514, no differences were detected between the predicted amino acid sequence generated by this construct and the corresponding wild type sequence of the Rst-IrreC protein.

\section{P-Element Transformation}

The basic protocol of Spradling and Rubin (1982) was used, with modifications described in Ramos et al. (1989) and Machado (1997). Constructs using pCaSpeR-hs as vector contain a white ${ }^{+}$minigene that partially rescues the white eye phenotype once integrated in the genome, so pCa18 $\Delta 3.1$ DNA, together with transposase-providing plasmid $\mathrm{p} \Delta 25.7 \mathrm{wc}$, was injected into $y w$ embryos before cellularization. Hatched larvae were allowed to develop into adulthood, surviving injected adults were separated in groups of 6-7 of the same sex, mated to 12-15 non injected $y w$ individuals and the progeny screened for the presence orange-reddish eyes.

GENETIC MOBILIZATION of $r s t-i r r e C^{p C a 18 Z \Delta 3.1}$ TRANSGENE

To generate autosomal insertions of the $\mathrm{P}\left[w^{+}\right.$ pCa18Z $\Delta 3.1]$ transgene, females genotypically

$$
y w P\left[w^{+} p C a 18 Z \Delta 3.1\right] / y w P\left[w^{+} p C a 18 Z \Delta 3.1\right] ;
$$

+/+ were crossed to +/Y; TM2 ry $\Delta 2-3 / \mathrm{TMS} S b$ $\Delta$ 2-3 males which constitutively express an activated form of the $\mathrm{P}$ element transposase. Orange eye males (carrying the transgene) that also showed stubble bristles (a dominant marker in the activated P-transposase chromosome) were selected from the progeny and crossed "en masse" with $y w$ virgin females. Since the $\mathrm{X}$ chromosome in the males is of maternal origin, any XY individual phenotypically non-white would perforce have the transgene transposed to an autosome. In four such events it was possible to generate stable stocks from the original male. In the cases were the TMS $S b \Delta 2-3$ was also present, it was removed by outcrossing before establishing the stock, in order to prevent further transposition events. Genetic mapping of the new transgene location was done by standard genetic methods (Greenspan 1997).

\section{RESULTS AND DISCUSSION}

Injection of DNA from construct pCa18 $\Delta 3.1$ in 1268 preblastoderm $y w$ embryos as described in Methods resulted in 66 surviving adults. One of these gave rise to a fraction of its progeny carrying orange eyes indicating germline integration of the transgene. The site of insertion was mapped genetically to chromosome $\mathrm{X}$ and a homozygous transgenic stock was established by inbreeding. Northern blots from PolyA ${ }^{+}$mRNA extracted from heat shocked embryos derived from this stock and probed with a rst-irre $C$ cDNA fragment, showed the presence of a $2 \mathrm{~kb}$ band, absent from controls without the transgene, and corresponding to the predicted size for the truncated rst-irreC mRNA produced by the pCa18Z 3.1 plasmid (Machado 1997). The transgenic flies were fertile and viable at $25^{\circ} \mathrm{C}$ both in heterozygous and homo- or hemizygous condition, but a dramatic decrease in embryonic hatching, compared to controls, was observed when transgenic embryos were allowed to develop at $30^{\circ} \mathrm{C}$ (Figure 1A). Also when individuals carrying the pCa $18 \Delta 3.1$ construct were subjected to heat-shock during late larval-early pupal stages the emerging adults showed a curled wing phenotype. This defect was sporadically observed when the transgenic stock was maintained at $25^{\circ} \mathrm{C}$ but never in the parental $y w$ flies independently of the temperature they were allowed to develop (Figure 1B-C).

These results suggested that overexpression of the extracellular domain alone of the Rst-irreC protein could interfere with developmental processes taking place in the embryo and in the wing primordia. However, given the fact that only one transgenic line was obtained, it could not be excluded 

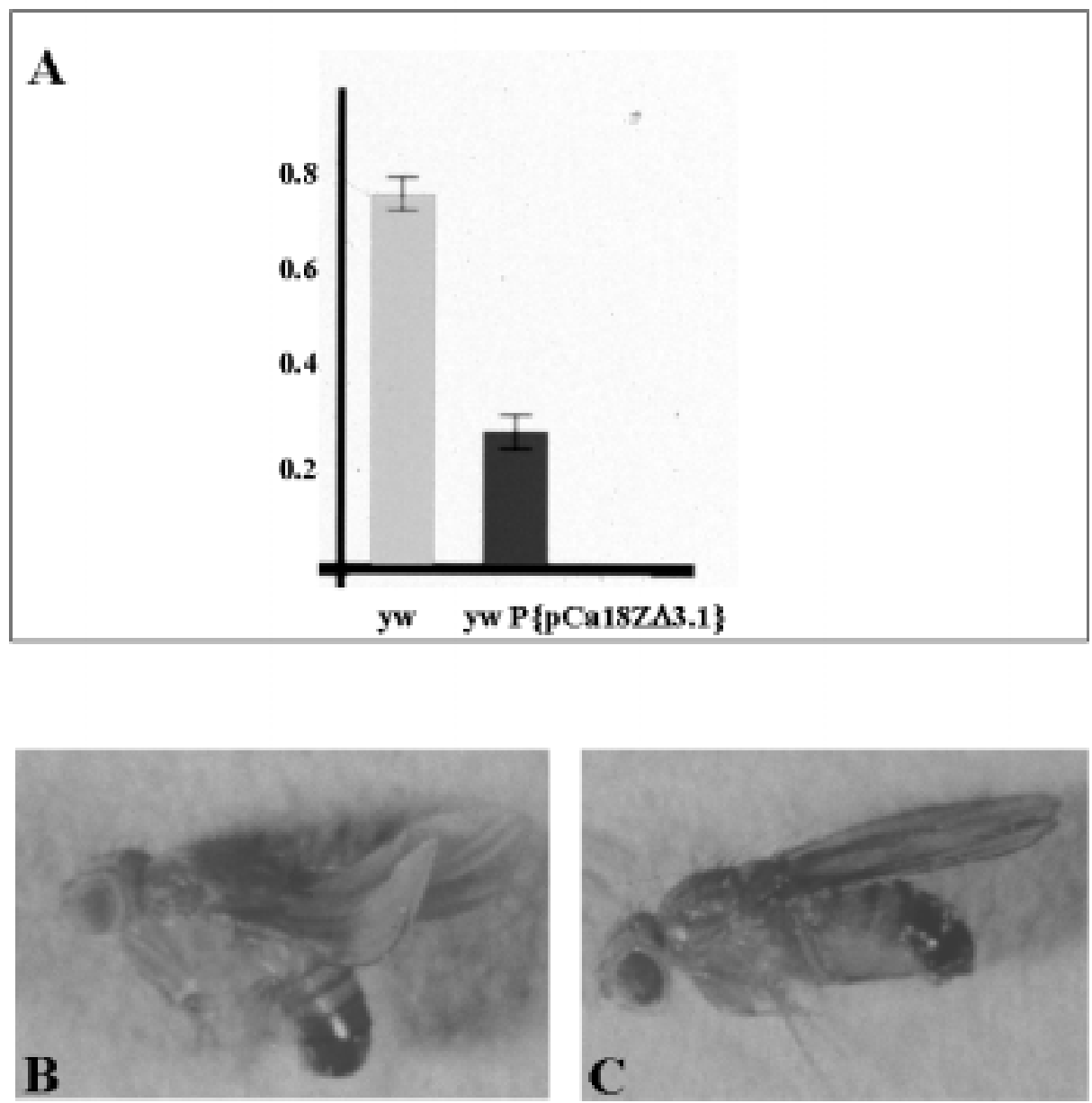

Fig. 1 - Phenotypic characterization of transgenic line pCa18 $\Delta 3.1$. (A) Viability of $0-4$ h embryos heat shocked for $24 \mathrm{hs}$ in percentage $\times 10^{-2}$. The parental strain $y w^{1118}$ was used as a control. Viability of the transgenic embryos at $25^{\circ} \mathrm{C}$ is about $85 \%$ of the parental strain (see figure 3). (B) Post embryonic curled wing phenotype of an transgene-carrying individual raised at $30^{\circ} \mathrm{C}$ from third instar larval stage to eclosion. (C) wild type fly.

that the observed phenotypes were caused by events independent of rst-irreC function, such as partial inactivation of a completely unrelated genetic function due to the insertion of the transgene into it. Also, the heat shock promoter present in $\mathrm{pCa} 18 \Delta 3.1$ could be interfering with the developmental regulation of genes adjacent to the site of insertion of the transgene. In order to rule out these possibilities we generated four additional transgenic lines, all autosomal, by genetically mobilizing the original pCa18Z $\Delta 3.1$ transgene. One of those, mapping in the second chromosome, turned out to be homozygous lethal even at $25^{\circ} \mathrm{C}$ and was not used in this study, but the other three, mapping in different regions of the third chromosome, showed good viability and fertility at this temperature and were kept as homozygous stocks. Although none of the mobilized lines showed the curled wing phenotype of the original transgenic stock, all showed a marked decrease in embryonic viability when grown at 29$30^{\circ} \mathrm{C}$ strongly suggesting that the latter phenotype was indeed linked to the transgene expression and 
A

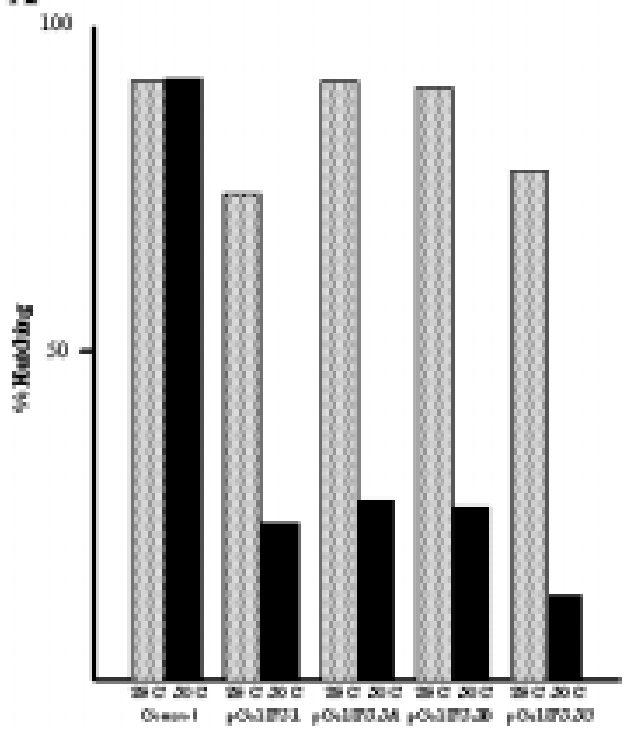

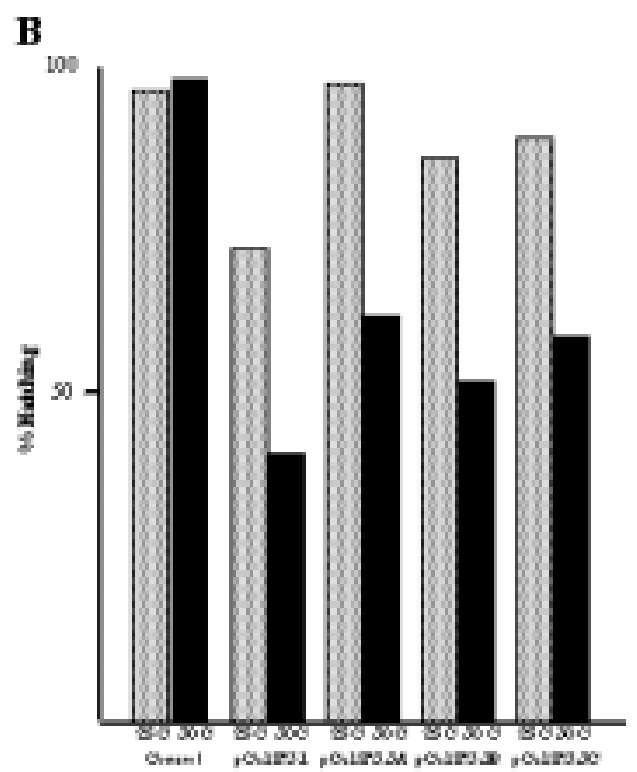

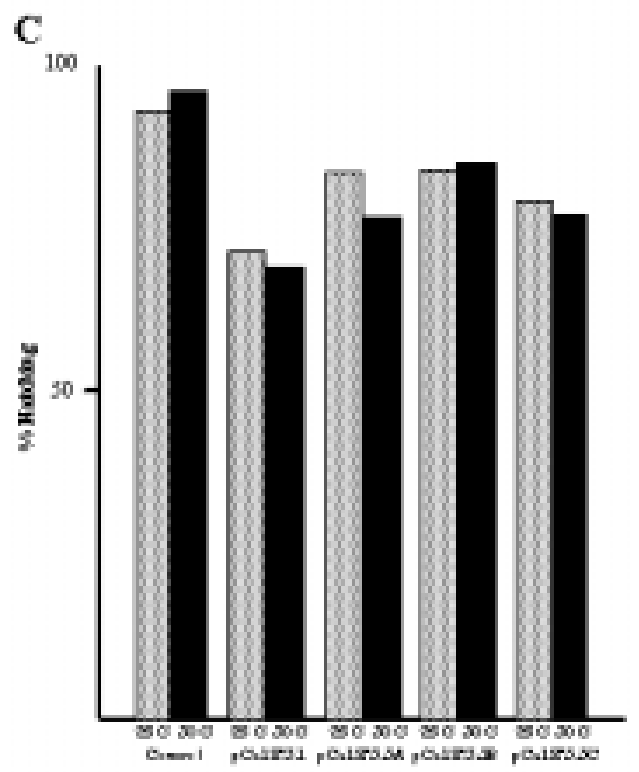

Fig. 2 - Percent hatching of embryos from the four pCa18 3.1 transgenic lines after being heat shocked in different embryonic stages as described in methods. (A) $0-2 \mathrm{~h}$ old embryos heat-shocked at $30^{\circ} \mathrm{C}$ for $8 \mathrm{hs}$. (B) 8-10hs old embryos heat shocked at $30^{\circ} \mathrm{C}$ for $8 \mathrm{hs}$. (C) 16-18h old embryos heat-shocked at $30^{\circ} \mathrm{C}$ for $8 \mathrm{hs}$.

not due to position dependent effects. We therefore tried to define when the administration of the heat shock could impair embryonic development, by examining the viability of embryos raised at $30^{\circ} \mathrm{C}$ between 0 -10hs, 8-18hs and 16-24hs of development (fig. 2A-C). Heat shocking embryos after $16 \mathrm{hs}$ of development seem to have little effect in their viability, but treatments at earlier time points clearly reduced the percentage of embryonic hatching, with the strongest and most consistent decrease being ob- 

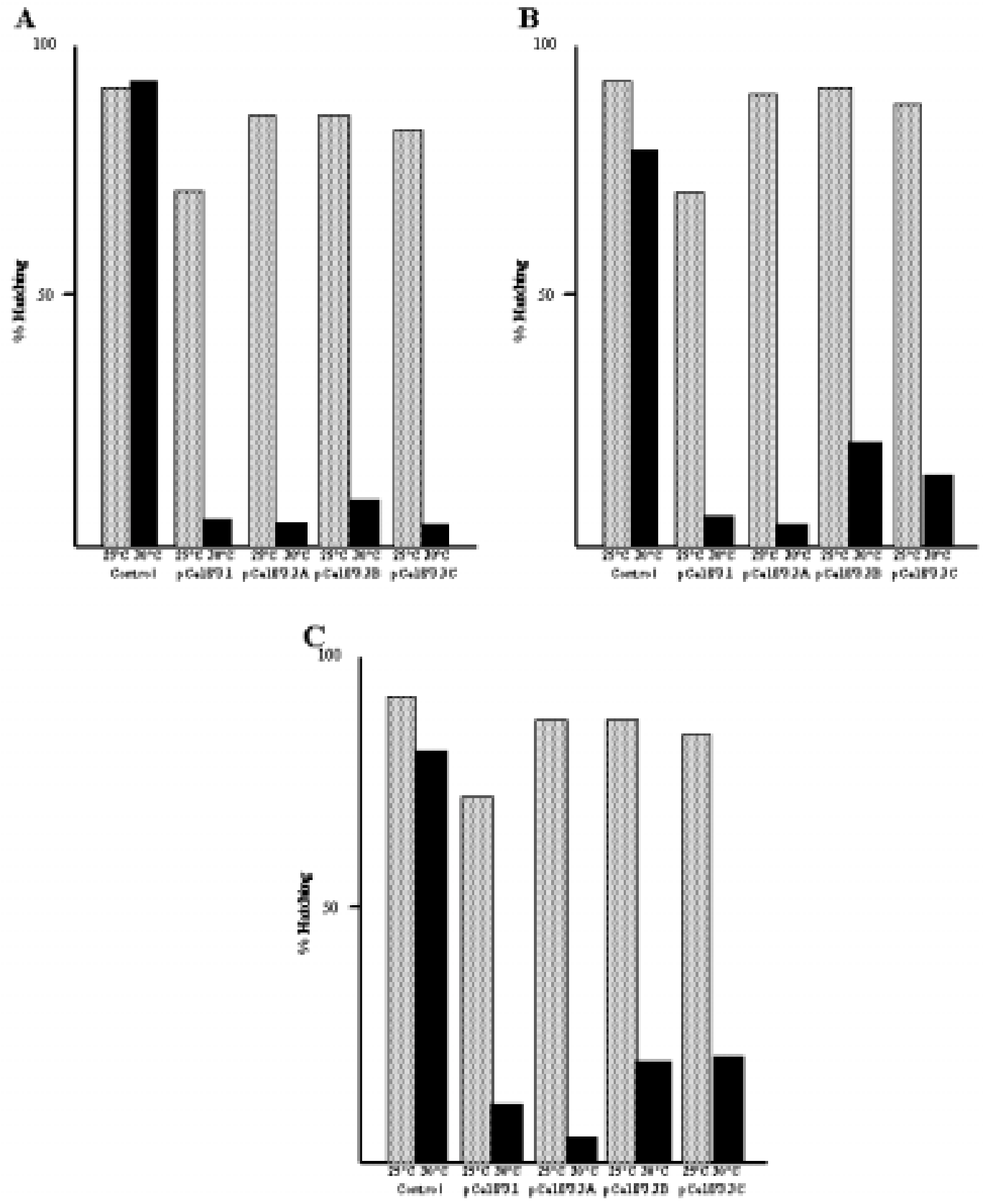

Fig. 3 - Effect of the length of the heat shock pulse in viability of embrios from the four pCa18 $\Delta 3.1$ transgenic lines. 0-1h old embryos were heat shocked for $2 \mathrm{hs}$ in (A), $4 \mathrm{hs}$ in (B) and $6 \mathrm{hs}$ in (C).

served when the heat pulse was applied during the first $10 \mathrm{hs}$ of development. Shorter heat shock pulses during the first 8 hours of development gave similar results (fig. 3A-C). Interestingly a two hour heat shock pulse in the first three hours of development, when endogenous Rst-IrreC is not yet present, seems to be sufficient to induce almost complete lethality (fig. 3C). Preliminary examination of the unhatched 
embryos indicates that in most of them development stopped shortly after the beginning of gastrulation (data not shown). Again the response of all four lines were similar, indicating that the site of insertion of the transgene is relatively unimportant as far as the embryonic lethality is concerned.

An important implication of our findings is the possibility that the observed embryonic phenotype could be due to heterophilic interactions of the extracellular domain: Previous experiments (Schneider et al. 1995) had shown that embryonic overexpression of full length rst-irre $C$ cDNA under heat shock control is also detrimental to normal development but since the product of the transgene was indistinguishable, structurally and functionally, from the endogenous wild type protein, it could not be determined whether the lethality was due to a homophilic interactions between ectopically expressed protein, to competition with the endogenous Rst-IrreC or by direct binding to a heterophilic partner. So far only homophilic binding has been directly demonstrated for the Rst-IrreC protein (Schneider et al. 1995). The extracellular domain encoded by pCa18Z $\Delta 3.1$ has neither an obvious membrane attachment site nor any other structural constraint that could in principle prevent if from being secreted into the extracellular space. Although we have not yet directly shown that the transgene-encoded polypeptide is secreted, an essentially identical construct under a different inducible promoter seems to be so (B. Bonengel, personal communication). Since lethality can be induced by expressing pCa18Z $\Delta 3.1$ in embryos at a developmental time (0-3hs) when wild type rstirre C mRNA is not yet detected by "in situ" hibridization (Ramos et al. 1993), it could be concluded that interactions between the transgenic protein and other cell surface or extracellular matrix molecules, rather than homophilic binding to endogenous RstIrreC are likely to be responsible for such reduction in viability. Additional support for this hypothesis comes from preliminary results recently obtained in our laboratory indicating that overexpression of the extracellular domain in a rst-irreC deficient mutant does not significantly attenuate the severity of the embryonic phenotype (L. Moda \& RGP Ramos, unpublished observations).

Finally it must be mentioned that the pCa18Z $\Delta 3.1$ protein product differs from the wild type Rst-IrreC extracellular domain by having a tyrosine to cysteine change at position 514 and 4 new amino acids at its carboxyl terminus. It cannot be excluded at the present that these differences are responsible for the embryonic lethality, although the fact tha similar results were obtained by overexpressing the complete protein (Schneider et al. 1995) strongly argues against this possibility. We are currently adressing this and other questions regarding the cellular and molecular basis of the this gain-of-function phenotype by expressing the extracellular domain in a more restricted pattern as well as characterizing in more detail the morphological defects associated with the lethal phenotype.

\section{ACKNOWLEDGEMENTS}

This paper is dedicated to the memory of Prof. Carlos Chagas Filho.

We thank Mara S.A. Costa for her expert technical assistance, Dr. Eliana Abdelhay for lab space in the initial phase of this work and Bernhard Bonengel for communicating unpublished results and for helpful discussions. This work was supported by grants from CNPq, FAPESP, and FAEPA.

\section{REFERENCES}

Boschert U, Ramos RGP, Tix S, Technau G \& FisСНвасн KF. 1990. Genetic and developmental analysis of irre $C$, a genetic function required for optic chiasm formation in Drosophila. J Neurogenetics 6: 153-171.

Elgin S \& Miller DM. 1980. Mass rearing of flies and mass production and harvesting of embryos. In: Ashburner M \& Wright TRF (Eds). The Genetics and Biology of Drosophila 2a: 112-121. New York. Academic Press.

GreEnSPAN RJ. 1997. Fly pushing: The theory and practice of Drosophila genetics. New York CSHL Press.

Lewis EB. 1960. A new standard food medium. 
Drosophila Inf Service 34: 117-118.

LindSLEy DC \& Zimm GG. 1992. The Genome of Drosophila Melanogaster San Diego, Academic Press 1133.

Machado RC. 1997. Análise funcional "in vivo" do locus irreC-rst de Drosophila melanogaster. Dissertação de mestrado 108pp. Instituto de Biofísica Carlos Chagas Filho da UFRJ, Rio de Janeiro.

Ramos RGP, Grimwade B, Wharton KA, Scotgale N \& Artavanis-Tsakonas S. 1989. "Physical and Functional Definition of the Drosophila Notch locus, by P-Element Transformation”. Genetics 123: 337348.

Ramos RGP, Igloi GL, Baumann, U, Maier D, Lichte B, Schneider T, Brandstätter JH, Fröhlich A \& Fischiach KF. 1993. "The irregular chiasm Croughest locus of Drosophila which affects axonal projections and programmed cell death encodes a novel immunoglobulin-like protein." Genes Dev 7: 2533-2547.
Reiter C, Schimansky T, Nie Z \& Fischbach KF. 1996. Reorganization of membrane contacts prior to apoptosis in the Drosophila retina: the role of the IrreC-rst protein. Development 122: 1931-1940.

Schneider T, Reiter C, Lichte B, Nie Z, Eule E, Bader B, Schimansky T, Ramos RGP \& Fischbach KF. 1995. Neural recognition in the optic lobe of Drosophila: restricted expression of the cell adhesion molecule IrreC-rst is required for normal axonal projections of columnar neurons. Neuron 15: 259271.

Spradling AC \& RUbin GM. 1982. Transposition of cloned P elements into Drosophila germline chromosomes. Science 218: 341-347.

Wolf T \& Ready DF. 1991. Cell death in normal and rough eye mutants of Drosophila. Development 113: 825-839. 